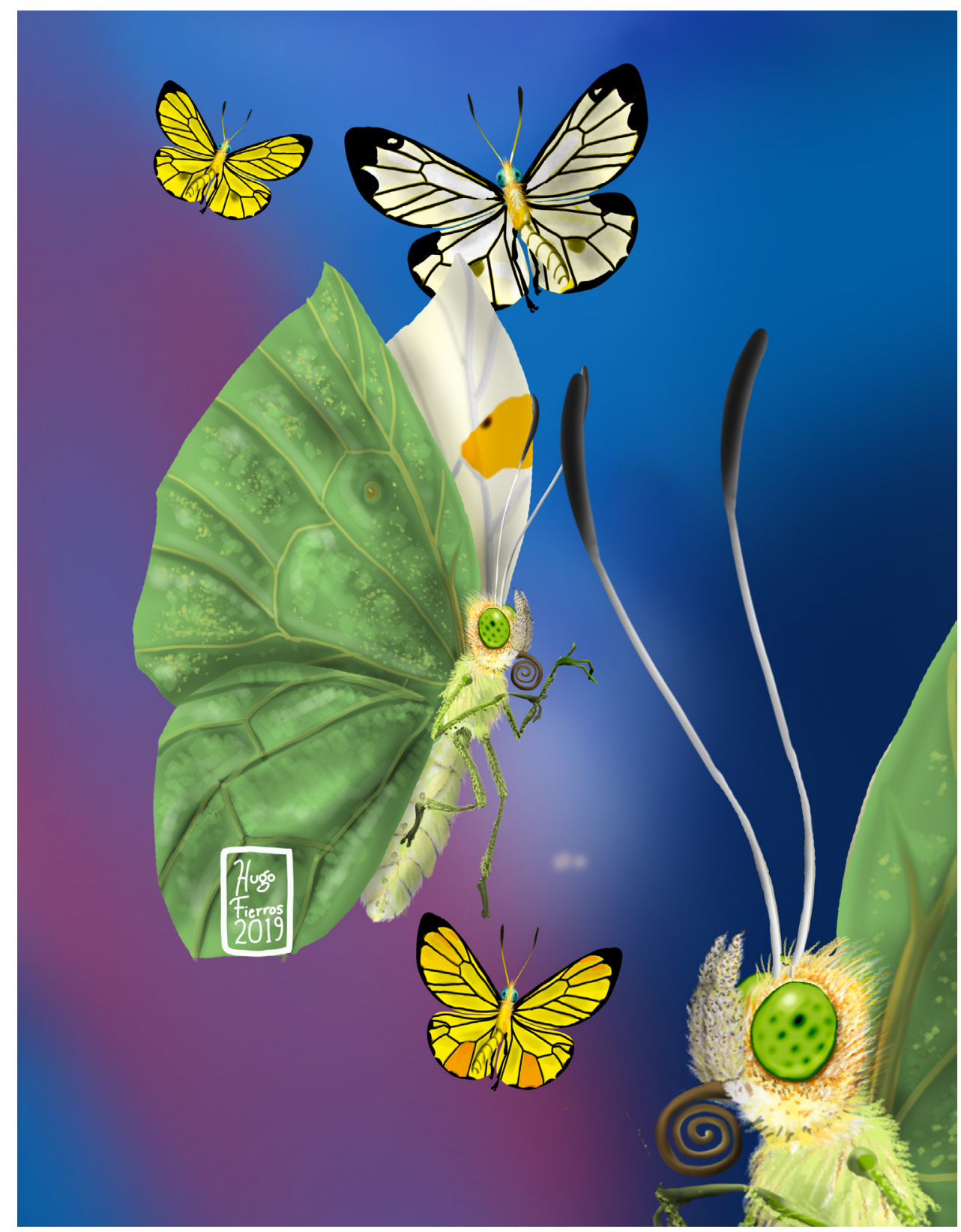

Dugesiana, Año 26, No. 2, julio 2019-diciembre 2019 (segundo semestre de 2019), es una publicación semestral, editada por la Universidad de Guadalajara, a través del Centro de Estudios en Zoología, por el Centro Universitario de Ciencias Biológicas y Agropecuarias. Camino Ramón Padilla Sánchez \# 2100, Nextipac, Zapopan, Jalisco, Tel. 37771150 ext. 33218, http://148.202.248.171/dugesiana/index.php/DUG/index, glenusmx@gmail.com. Editor responsable: José Luis Navarrete Heredia. Reserva de Derechos al Uso Exclusivo 04-2009-062310115100203, ISSN: 2007-9133, otorgados por el Instituto Nacional del Derecho de Autor. Responsable de la última actualización de este número: José Luis Navarrete Heredia, Editor y Ana Laura González-Hernández, Asistente Editorial. Fecha de la última modificación 25 de julio 2019, con un tiraje de un ejemplar.

Las opiniones expresadas por los autores no necesariamente reflejan la postura del editor de la publicación.

Queda estrictamente prohibida la reproducción total o parcial de los contenidos e imágenes de la publicación sin previa autorización de la Universidad de Guadalajara. 
Artículo

\title{
Ciempiés de Sinaloa, México (Myriapoda: Chilopoda): nuevos registros de especies y ampliación de su distribución estatal
}

\section{Centipedes of Sinaloa, Mexico (Myriapoda: Chilopoda): new species records and expansion of its regional distribution}

\author{
Fernando López-Bonel ${ }^{1}$, Claudia Patricia Ávila-Gaxiola ${ }^{1}$ y Fabio G. Cupul-Magaña ${ }^{2 *}$ \\ ${ }^{1}$ Egresado de la licenciatura en Biología de la Universidad Autónoma de Sinaloa, Blvd. Miguel Tamayo Espinoza \\ de los Monteros 2358, Desarrollo Urbano 3 Ríos, 80020, Culiacán de Rosales, Sinaloa. México, est.fernando. \\ lopez@uas.edu.mx,patyag_1@hotmail.com; 르르o Universitario de la Costa, Universidad de Guadalajara, Av. \\ Universidad 203, Delegación Ixtapa, 48280, Puerto Vallarta, Jalisco, México, fabiocupul@gmail.com.
}

\section{RESUMEN}

Se han registrado siete especies y una subespecie de ciempiés en Sinaloa. El estudio de nuevo material, recolectado de 2016 a 2018, permitió identificar 12 especies de ciempiés, de las cuales ocho representan nuevos registros estatales. Las otras cuatro especies, previamente documentadas para el estado, son registradas en nuevas localidades. Presentamos una lista con los nuevos registros para el estado de Dendrothereua linceci (Wood, 1867), Scolopendra morsitants Linnaeus, 1758, Newportia quadrimeropus (Shelley \& Mercurio, 2005), N. stolli (Pocock, 1896), Pachymerium ferrugineum (Koch, 1835), Mecistocephalus guildingii Newport, 1843, Notiphilides maximiliani (Humbert \& Saussure, 1870) y Orphnaeus brevilabiatus (Newport,1845), así como nuevos registros de localidades para Arthrorhabdus pygmaeus (Pocock, 1895), S. polymorpha Wood, 1861, S. viridis Say, 1821 y Rhysida longipes (Newport, 1845). La lista actualizada de especies de ciempiés para Sinaloa es de 15 especies y una subespecie. En México, el estado está clasificado octavo en riqueza de ciempiés.

Palabras clave: distribución, Geophilomorpha, lista, riqueza, Scolopendromorpha, Scutigeromorpha.

\section{ABSTRACT}

Seven species and one subspecies of centipedes have been recorded in Sinaloa. The study of new specimens, collected from 2016 to 2018, identified 12 centipedes species, eigth of them are new state records. The other four species, previously recorded in the state, were registered in new localities. We present a list with the new state records of Dendrothereua linceci (Wood, 1867), Scolopendra morsitants Linnaeus, 1758, Newportia quadrimeropus (Shelley \& Mercurio, 2005), N. stolli (Pocock, 1896), Pachymerium ferrugineum (Koch, 1835), Mecistocephalus guildingii Newport, 1843, Notiphilides maximiliani (Humbert \& Saussure, 1870) and Orphnaeus brevilabiatus (Newport,1845), and new localities records from Arthrorhabdus pygmaeus (Pocock, 1895), S. polymorpha Wood, 1861, S. viridis Say, 1821 and Rhysida longipes (Newport, 1845). The current checklist of the Sinaloa state centipedes includes 15 species and one subspecies. In Mexico, the state is ranked eigth in centipedes richness.

Key words: checklist, distribution, Geophilomorpha, richness, Scolopendromorpha, Scutigeromorpha.

La clase Chilopoda agrupa cinco órdenes (Scutigeromorpha, Lithobiomorpha, Craterostigmomorpha, Scolopendromorpha y Geophilomorpha) que, de acuerdo con Bonato et al. (2016) incluyen 3,235 especies válidas de artrópodos terrestres conocidos comúnmente como ciempiés. Su cuerpo se divide en cabeza y tronco, este último multisegmentado y con un par de apéndices por segmento (Ribera et al. 2015).

Los quilópodos pueden medir desde unos cuantos milímetros hasta $30 \mathrm{~cm}$ de longitud, así como presentar de 15 a 191 pares de patas en los especímenes adultos (Minelli 2011). En la cabeza tienen un par de forcípulas con la capacidad de inocular veneno (Eisner et al. 2005). Son depredadores que en su dieta incluyen lombrices, artrópodos, así como pequeños reptiles, anfibios y mamíferos (Lewis 1981; Molinari et al. 2005; Voigtländer 2011; Folly et al. 2019). La humedad es el factor abiótico más importante que influye sobre su distribución geográfica (Voigtländer 2011).

En México se han registrado 183 especies de ciempiés
(143 endémicas, 78.14\%) en 14 familias, que están incluidos en cuatro de los cinco órdenes existentes a nivel mundial (Cupul-Magaña 2019): Scutigeromorpha (seis especies), Lithobiomorpha (59 especies), Scolopendromorpha (43 especies) y Geophilomorpha (75 especies).

Los datos sobre su distribución geográfica en el país indican un mayor registro de especies para la región centro, sur, noreste y oriente; además, Tlaxcala es el único estado sin reportes históricos (Cupul-Magaña 2013, 2019). Para el estado de Sinaloa, aunque se tienen estudios sobre la diversidad de ciempiés (Ávila-Gaxiola et al. 2017; CortésRíos y Gárate-Rodríguez 2017; Beltrán-García 2018), solo se conocen siete especies y una subespecie, dos de ellas y la subespecie son endémicas del país y el resto son de amplia distribución (Cupul-Magaña 2014).

El objetivo de este trabajo es documentar nuevos registros de localidades y de especies de ciempiés para Sinaloa, a partir de material recolectado en campo en los municipios de Badiraguato, Culiacán, Navolato, Elota y Concordia. 


\section{MATERIAL Y MÉTODOS}

El material miriapodológico provine de recolectas no sistemáticas realizadas durante los años 2016, 2017 y 2018 en cinco de los 18 municipios del estado de Sinaloa (coordenadas extremas $22^{\circ} 31^{\prime}-26^{\circ} 56^{\prime} \mathrm{N}$ y $105^{\circ} 24^{\prime}-$ $109^{\circ} 27^{\prime}$ O; Fig. 1), principalmente en los ubicados en la zona centro y sur. Los ejemplares se recolectaron de manera directa de entre la hojarasca, bajo troncos y rocas. Se preservaron en alcohol al $96 \%$ y se depositaron en la colección zoológica de la Escuela de Biología de la Universidad Autónoma de Sinaloa en Culiacán.

Para la identificación de los ciempiés hasta el nivel de especie, se utilizaron los trabajos de Verhoeff (1934), Würmli (1973), Shelley (2002), Bonato et al. (2004), Shelley y Mercurio (2005), Edgecombe y Cupul-Magaña (2008), Schileyko (2013), así como Calvanese y Brescovit (2017). A cada especie identificada se le asociaron datos del material examinado y de recolecta, así como información sobre su distribución geográfica y registros previos en Sinaloa. Cuando el sexo de los ejemplares no fue identificado se señaló como snd. Para designar al colector o los colectores se usó la abreviatura col.

\section{RESULTADOS Y DISCUSIÓN}

Se identificaron 12 especies de ciempiés: orden Scutigeromorpha (una especie), orden Scolopendromorpha (siete especies) y orden Geophilomorpha (cuatro especies). Además, aunque en el trabajo de campo también se recolectó material biológico de los órdenes Lithobiomorpha y Geophilomorpha, no se incluyó en los resultados; puesto que se identificó solo a nivel de morfoespecie, con al menos seis en cada orden. Para su posterior identificación, será importante revisar detalladamente las descripciones de las especies presentes en México y realizar las comparaciones con el material tipo depositado en diversas colecciones; ya que un número significativo de las descripciones originales de la fauna de ciempiés del país son escuetas y carecen de ilustraciones, lo que dificulta su empleo como guías precisas de determinación taxonómica (Mundel 1981; Cupul-Magaña 2013).

Las especies Aztekophilus mexicanus Verhoeff, 1934, Scolopendra heros Girard, 1853, S. octodentata Verhoeff, 1934 y S. viridis genuina Verhoeff, 1934, previamente reportadas para Sinaloa (Verhoeff 1934; Shelley 2002; Cupul-Magaña 2014), no se encontraron en las muestras revisadas en este trabajo; seguramente porque no se incluyeron los mismos sitios donde se les había registrado: Topolobampo en Ahome y áreas aledañas a las zonas urbanas de Culiacán y Mazatlán (Cupul-Magaña 2014). Sin embargo, las restantes cuatro especies conocidas para el estado se registraron para nuevas localidades. Ocho de las 12 especies recolectadas en este estudio son nuevos registros para la entidad. Así, el número actual de especies de ciempiés para Sinaloa es de 15 más una subespecie.

Este número de especies ubica a Sinaloa en el octavo lugar de riqueza de ciempiés en el país, empatado con Jalisco y Oaxaca, y superado por Veracruz y Nuevo León, los dos estados más diversos con 43 y 23 especies, respectivamente (Cupul-Magaña 2013). No obstante, aunque entre los factores básicos que rigen la distribución geográfica de los ciempiés, y de otros animales terrestres, están la historia geológica y el clima (Mitić y Tomić 2002); es posible afirmar que la distribución de la quilopodofauna en el país es en parte resultado de un sesgo en las áreas exploradas para la recolecta de ejemplares durante casi dos siglos (Bonato y Zapparolii 2011; el registro histórico de las exploraciones en México puede consultarse en CupulMagaña 2013), por lo que puede esperarse que un aumento en las campañas de búsqueda en campo en Sinaloa, incremente sustancialmente su riqueza.

A pesar de que Cortés-Ríos y Gárate-Rodríguez (2017), así como Beltrán-García (2018), mencionan la presencia en el estado de Clinopodes flavidus (Koch, 1847), Lithobius forficatus (Linnaeus, 1758), Scolopendra pomacea Koch, 1847 y Scutigera coleoptrata (Linnaeus, 1758), estas especies deben ser omitidas de la lista hasta su validación. La duda sobre la identidad correcta de los ejemplares, surge porque los trabajos que los autores citan como base para la determinación de los especímenes examinados, son listas de especies y otros solo permiten la identificación hasta nivel de familia, pero no hasta especie. Además, aunque los autores mencionan que recibieron ayuda de especialistas, no puntualizan sobre las especies que estos determinaron.

Hasta el momento, los ciempiés que habitan Sinaloa corresponden a especies con gran habilidad de dispersión (géneros Dendrothereua, Mecistocephalus, Pachymerium), de presencia en ambientes tropicales (géneros Arthrorhabdus, Newportia, Notiphilides, Orphnaeus, Scolopendra, Rhysida) y templados (géneros Scolopendra), así como endémicas ( $S$. octodentata y $S$. viridis genuina) (Bonato 2011; Bonato y Zapparolli 2011; Edgecombe y Bonato 2011). De hecho, el estado y México en general, se encuentran en una de las regiones más ricas de fauna de ciempiés del mundo (Bonato y Zapparolli 2011), seguramente como consecuencia de una variada historia biogeográfica que ha dado como resultado una gradación de climas que abarcan una zona Neárica en el norte y una Neotropical en el sur (Ramamoorthy et al. 1998). Por lo anterior, el paso siguiente a este tipo de inventarios de la quilopodofauna es estudiar la ecología de las especies presentes.

A continuación, se presenta la lista de las especies registradas:

Chilopoda Latreille, 1817

Scutigeromorpha Pocock, 1895

Scutigeridae Leach, 1814

Scutigerinae Leach, 1814

Dendrothereua linceci (Wood, 1867)

Localidades de recolecta: El Palmito, Concordia; 233' 16.2” N, 105'51'00.7” O; dos machos; 26/X/2016; recolecta diurna directa sobre tronco podrido; bosque de pino; col. C.P. Ávila-Gaxiola y F. López-Bonel. $58 \mathrm{~km}$ al norte de Badiraguato con dirección a Surutato; 25 43'41.9" N, 107033'50.1" O; un macho y una hembra; 20/X/2017; recolecta diurna directa bajo roca y en hojarasca; bosque de encino; col. F. López-Bonel. Surutato, Badiraguato; 25'49'47.3" N, 107³3' 49.0" O; dos machos; 17/III/2018; recolecta diurna directa sobre tronco podrido; bosque de pino; col. F. López-Bonel. Chirimole, Elota; $24^{\circ} 15^{\prime} 11.4^{\prime \prime} \mathrm{N}$, 106 39'20.8" O; dos machos; 23/VI/2018; recolecta directa diurna bajo roca y en orilla de río; selva baja subcaducifolia; col. F. López-Bonel (Fig. 2).

Distribución: desde el sur de los Estados Unidos de 
América, México, Centroamérica y el Caribe (Edgecombe y Cupul-Magaña 2008; Bonato et al. 2016).

Distribución en México: Baja California Sur, Ciudad de México, Chiapas, Chihuahua, Guerrero, Hidalgo, Jalisco, Michoacán, Morelos, Oaxaca, Puebla, Quintana Roo, San Luis Potosí, Tabasco y Veracruz (Edgecombe y Cupul-Magaña 2008; Cupul-Magaña et al. 2015; FloresGuerrero et al. 2015; Cupul-Magaña y Bueno-Villegas 2016).

Registros en Sinaloa: nuevo registro de la especie para el estado.

Scolopendromorpha Pocock, 1895

Scolopendridae Leach, 1814

Scolopendrinae Leach, 1814

Arthrorhabdus pygmaeus (Pocock, 1895)

Localidades de recolecta: $16 \mathrm{~km}$ al noroeste de Culiacán con dirección a Imala; 24'52'44.3" $\mathrm{N}$, $107^{\circ} 16^{\prime} 44.3$ " O, altitud $37 \mathrm{~m}$; un snd; 24/X/2017; recolecta diurna directa bajo roca y a una temperatura ambiental de $31{ }^{\circ} \mathrm{C}$; selva baja caducifolia con predominio del vinolo (Acacia cochliacantha Humb. \& Bonpl. ex Willd.); col. F. López-Bonel (Fig. 3). (Nueva localidad de distribución en Sinaloa).

Distribución: Estados Unidos de América y México; probablemente en Belice (Shelley y Chagas-Jr 2004; Bonato et al. 2016).

Distribución en México: Baja California Sur, Coahuila, Colima, Durango, Guerrero, Hidalgo, Jalisco, Morelos, Nayarit, Nuevo León, Oaxaca, Puebla, San Luis Potosí, Sinaloa, Tamaulipas y Quintana Roo (Shelley 2002; CupulMagaña et al. 2015; Flores-Guerrero et al. 2015).

Registros en Sinaloa: $9.6 \mathrm{~km}$ y $64 \mathrm{~km}$ al sur de Culiacán; Mazatlán (Shelley 2002; Shelley y Chagas-Jr 2004; Fig. 3).

Scolopendra morsitants Linnaeus, 1758

Localidades de recolecta: Agua Blanca, Culiacán;

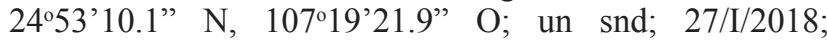
recolecta diurna directa bajo tronco y hojarasca; selva baja caducifolia con presencia de casiguano [Caesalpinia eriostachys (Benth.) Britton \& Rose], amapa rosa [Handroanthus impetiginosus (Mart. ex DC.) Standl.] y vinolo; col. C. P. Ávila-Gaxiola (Fig. 4).

Distribución: Estados Unidos de América, México, Centro y Sudamérica, el Caribe, África, Asia, Australia, Islas del Pacífico, India y Pacífico occidental y central; con frecuencia introducida (Shelley et al. 2005; Shelley 2006; Bonato et al. 2016).

Distribución en México: Baja California Sur, Ciudad de México, Coahuila, Colima, Estado de México, Guerrero, Jalisco, Morelos, Oaxaca, Puebla, Querétaro, Tamaulipas, Veracruz y Yucatán (Shelley 2002, 2006; Cupul-Magaña 2013; Cupul-Magaña et al. 2015; Flores-Guerrero et al. 2015).

Registros en Sinaloa: nuevo registro de la especie para el estado.

Scolopendra polymorpha Wood, 1861

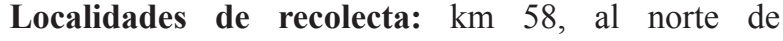
Badiraguato con dirección a Surutato; 25\%43'41.9" N, 107³3'50.1" O, altitud 1,292 m; dos snd; 2017; zona perturbada por carretera; recolecta diurna directa dentro de tronco podrido; bosque de encino; col. F. López-Bonel (Fig. 5). (Nueva localidad de distribución en Sinaloa).

Distribución: Estados Unidos de América y México; posiblemente Belice e introducida en Hawái (Shelley 2006).

Distribución en México: Baja California, Baja California Sur, Chihuahua, Coahuila, Estado de México, Durango, Guerrero, Hidalgo, Jalisco, Michoacán, Morelos, Nuevo León, Oaxaca, Puebla, Querétaro, Sinaloa, Sonora, Tamaulipas y Zacatecas (Shelley 2002; Cupul-Magaña 2013; Tabullo-De Robles et al. 2014).

Registros en Sinaloa: El Fuerte; El Varejonal, municipio Badiraguato; Presa Sanalona, carretera a Tamazula, municipio de Culiacán (Cupul-Magaña 2013; Flores-Guerrero et al. 2015; Fig. 5).

\section{Scolopendra viridis Say, 1861}

Localidades de recolecta: Instalaciones del Centro de Estudios Justo Sierra (CEJUS), Surutato, Badiraguato; 25'49'44.3" N, 107'33'53.3" O, altitud 1,534 m; un snd, 20/X/2017; un snd, 22/X/2017; tres snd, 17/XI/2017; recolecta diurna directa bajo rocas; col. F. López-Bonel. $\mathrm{Km}$ 58, al norte de Badiraguato con dirección a Surutato; 2543'41.9" N, 107³3'50.1" O; dos snd; 2017; zona perturbada por carretera (Fig. 6). (Nuevas localidades de distribución en Sinaloa).

Distribución: Costa Rica, El Salvador, Estados Unidos de América, Guatemala, Honduras, Nicaragua, Panamá y México (Shelley 2002; Bonato et al. 2016).

Distribución en México: Aguascalientes, Baja California, Baja California Sur, Ciudad de México, Chihuahua, Chiapas, Coahuila, Colima, Durango, Estado de México, Guerrero, Guanajuato, Hidalgo, Jalisco, Michoacán, Morelos, Nayarit, Nuevo León, Oaxaca, Puebla, Querétaro, San Luis Potosí, Sinaloa, Sonora, Tamaulipas, Veracruz y Zacatecas (Shelley 2002; CupulMagaña 2013).

Registros en Sinaloa: $3.2 \mathrm{~km}$ al noroeste de Choix; $32 \mathrm{~km}$ al este de Concordia; $9.6 \mathrm{~km}$ al sur de Guamúchil; $83.2 \mathrm{~km}$ al este de Villa Unión; Topolobampo, municipio de Ahome (Verhoeff 1934; Shelley 2002; Flores-Guerrero et al. 2015; Fig. 6).

Otostigminae Kraepelin, 1903

Rhysida longipes (Newport, 1845)

Localidades de recolecta: sin localidad específica, Navolato; un snd; 12/XI/2016; recolecta diurna directa bajo rocas de jardín; col. C. P. Ávila-Gaxiola. Colonia Villa Universidad, Culiacán; 2449'55.6” N, 107²2'52.4" O; un snd; 10/V/2017; bajo roca en lote baldío con vegetación secundaria de ricino (Ricinus communis L.) y amaranto espinoso (Amaranthus spinosus L.); col. F. López-Bonel.

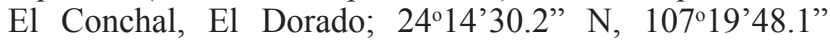
O; dos snd; 9/VIII/2017; recolecta diurna directa bajo macetas; col. F. López-Bonel. Departamento de Curaduría, Jardín Botánico, Culiacán; 2449’25.5” N, 107²3'09.0” O; tres snd, 9/IV/2018; un snd, 30/IV/2018; recolecta diurna entre la hojarasca; col. F. López-Bonel (Fig. 7). (Nuevas localidades de distribución en Sinaloa).

Distribución: de amplia distribución mundial (ChagasJr 2013; Bonato et al. 2016) 
Distribución en México: Campeche, Jalisco, Michoacán, Morelos, Nayarit y Sinaloa (Cupul-Magaña, 2013; Tabullo-De Robles et al. 2014).

Registros en Sinaloa: Mazatlán y zona urbana de Los Mochis (Pocock 1895-1910; Cupul-Magaña 2009; FloresGuerrero et al. 2015; Fig. 7).

Scolopocryptopidae Pocock, 1896

Newportiinae Pocock, 1896

Newportia (Ectonocryptoides) quadrimeropus (Shelley \& Mercurio, 2005)

Localidades de recolecta: $2 \mathrm{~km}$ al norte de la localidad

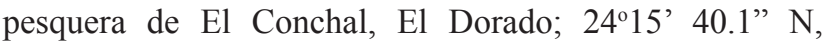
107'20'15.3" O; un snd; 28/VIII/2017; recolecta diurna directa entre la hojarasca; selva baja caducifolia; col. F. López-Bonel (Fig. 8).

Distribución: México (Shelley y Mercurio 2005; Shelley 2009; Cupul-Magaña y Shelley 2011).

Distribución en México: Jalisco y Puebla (Shelley y Mercurio 2005; Shelley 2009; Cupul-Magaña y Shelley 2011).

Registros en Sinaloa: nuevo registro de la especie para el estado.

Newportia stolli (Pocock, 1896)

Localidades de recolecta: Jardín Botánico, área de colección de plantas de la selva tropical, Culiacán; 2449'22.7” N, 107'23'08.0" O; dos snd, 16/II/2018; un snd, 9/IV/2018; recolecta diurna directa entre la hojarasca; col. F. López-Bonel (Fig. 9).

Distribución: Belice, Brasil, Colombia, Cuba, Guatemala, Honduras y México (Schileyko y Minelli 1998; Schileyko 2002; Martínez-Muñoz y Tcherva 2017).

Distribución en México: Campeche, Chiapas, Morelos y Veracruz (Chagas-Jr y Shelley 2003; Flores-Guerrero et al. 2015).

Registros en Sinaloa: nuevo registro de la especie para el estado.

Geophilomorpha Pocock, 1895 Geophilidae Leach, 1815

Pachymeriinae Verhoeff, 1925

Pachymerium ferrugineum (Koch, 1835)

Localidades de recolecta: Parque Riberas, Culiacán;

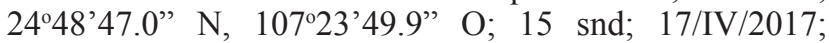
recolecta diurna directa en tronco húmedo a la orilla del río; bosque de galería con presencia de sauce llorón (Salix babylonica L.); col. F. López-Bonel. Río Culiacán, 23 $\mathrm{km}$ al suroeste de Culiacán con dirección a Navolato; un snd; 13/X/2017; recolecta diurna directa en la hojarasca; bosque de galería con presencia de $S$. babylonica; col. F. López-Bonel. Agua Blanca, Culiacán; 2453'10.1” N, $107^{\circ} 19^{\prime} 21.9^{\prime \prime} \mathrm{O}$; un snd; 27/I/2018; recolecta diurna directa en la hojarasca; selva baja caducifolia con presencia de $A$. cochliacantha, C. eriostachys y H. impetiginosus; col. F. López-Bonel. Centro de Estudios Justo Sierra (CEJUS), Surutato, Badiraguato; 2549'47.3" N, 107'33'49.0" O; cuatro snd, $22 / \mathrm{X} / 2017 ; 25^{\circ} 49^{\prime} 44.3^{\prime \prime} \mathrm{N}, 107^{\circ} 33^{\prime} 53.3$ " O; tres snd, 20/V/2018; recolecta diurna directa en la hojarasca; bosque de pino; col. F. López-Bonel (Fig. 10).

Distribución: especie transpaleártica polizonal (ampliamente distribuida en tundra, taiga y otras regiones) e introducida en Norte y Sudamérica, Japón y Hawái (Dyachkov 2018).

Distribución en México: Baja California Sur, Nayarit y Veracruz (Verhoeff 1934; Cupul-Magaña 2015; FloresGuerrero et al. 2015).

Registros en Sinaloa: nuevo registro de la especie para el estado.

Mecistocephalidae Bollman, 1893

Mecistocephalinae Verhoeff, 1901

Mecistocephalus guildingii Newport, 1843

Localidades de recolecta: Jardín Botánico, área de colección de plantas de la selva tropical, Culiacán; 244'22.7” N, 107²3'08.0” O; dos snd, 9/X/2017; 5 snd,16/II/2018; un snd, 30/IV/2018; recolecta diurna directa bajo rocas; col. F. López-Bonel (Fig. 11).

Distribución: sureste de los Estados Unidos de América, México, el Caribe, Panamá, Brasil, norte de Europa, oeste de África (Bonato et al. 2009).

Distribución en México: Jalisco (Bonato et al. 2009).

Registros en Sinaloa: nuevo registro de la especie para el estado.

Oryidae Cook, 1895

Oryinae Attems, 1914

Notiphilides maximiliani (Humbert \& Saussure, 1870)

Localidades de recolecta: en viveros del Jardín Botánico, Culiacán; 2449'25.3” N, 107²3'12.1” O; cuatro snd; 16/II/2018; recolecta diurna directa bajo macetas, troncos y rocas; col. F. López-Bonel (Fig. 12).

Distribución: Barbados, Colombia, Dominica, Ecuador, Granada, Guatemala, Martinica, Perú, Santa Lucia, San Vicente, Trinidad y Tobago, así como Venezuela (Bonato et al. 2016).

Distribución en México: Campeche, Tabasco y Yucatán (Cupul-Magaña 2013).

Registros en Sinaloa: nuevo registro de la especie para el estado.

Orphnaeus brevilabiatus (Newport,1845)

Localidades de recolecta: El Palmito, Concordia; 23034'16.2" N, 105'51"00.7" O; un macho; 26/X/2016; recolecta diurna directa en tronco podrido; bosque de pino; col. C.P. Ávila-Gaxiola y F. López-Bonel (Fig. 13).

Distribución: amplia distribución en regiones tropicales, a menudo introducida (Bonato 2011).

Distribución en México: Jalisco y Tamaulipas (CupulMagaña 2013).

Registros en Sinaloa: nuevo registro de la especie para el estado.

\section{AGRADECIMIENTOS}

A la dirección de la Facultad de Biología de la Universidad Autónoma de Sinaloa y al Dr. Víctor Manuel Salomón Soto por las facilidades otorgadas para la realización del trabajo de campo. A los revisores anónimos por sus comentarios. Trabajo dedicado al Dr. Jorge Llorente Bousquets de la Facultad de Ciencias de la UNAM. 


\section{LITERATURA CITADA}

Ávila-Gaxiola, C.P., F. López-Bonel y J. López-de Dios, J. 2017. Diversidad de miriápodos en ecosistema de manglar en El Conchal, Sinaloa. Boletín de la Sociedad Mexicana de Entomología (n. s.), (3): 7-11.

Beltrán-García, L.C. 2018. Diversidad de miriápodos (Diplopoda y Chilopoda) en dos localidades de San Ignacio, Sinaloa. Boletín de la Sociedad Mexicana de Entomología (n. s.), (4): 1-4.

Bonato, L. 2011. Order Geophilomorpha. (pp. 407-443). In: Minelli, A. (Ed.). The Myriapoda: Treatise on zoology - anatomy, taxonomy, biology. Volume 1. Brill, Leiden.

Bonato, L. and M. Zapparolli. 2011. Chilopoda Geographical distribution. (pp. 327-337). In: Minelli, A. (Ed.). The Myriapoda: Treatise on zoology - anatomy, taxonomy, biology. Volume 1. Brill, Leiden.

Bonato, L., D. Foddai and A. Minelli. 2004. The centipede order Geophilomorpha in the Hawaiian Islands (Chilopoda). Records of the Hawaii Biological Survey for 2003. Bishop Museum Occasional Papers, (78): 1332.

Bonato, L., F.G. Cupul-Magaña and A. Minelli. 2009. Mecistocephalus guildingii Newport, 1843, a tropical centipede with amphi-Atlantic distribution (Chilopoda: Geophilomorpha). Zootaxa, (2271): 27-42.

Bonato L., A. Chagas-Jr, G.D. Edgecombe, J.G.E. Lewis, A. Minelli, L.A. Pereira, R.M. Shelley, P. Stoev and M. Zapparoli. 2016. ChiloBase 2.0 - A World Catalogue of Centipedes (Chilopoda). http://chilobase.biologia. unipd.it. Fecha de consulta: 15 de enero de 2019.

Calvanese, V.C. and A.D. Brescovit. 2017. A new species of Notiphilides from Amazonia, with a redescription of $N$. grandis (Chilopoda, Geophilomorpha, Oryidae). Zootaxa, 4232(1): 1-20.

Chagas-Jr, A. 2013. A redescription of Rhysida celeris (Humbert \& Saussure, 1870), with a proposal of eight new synonyms (Scolopendromorpha, Scolopendridae, Otostigminae). ZooKeys, (258): 17-29.

Chagas-Jr, A. and R.M. Shelley. 2003. The centipede genus Newportia Gervais, 1847, in Mexico: description of a new troglomorphic species; redescription of N. sabina Chamberlin, 1942; revival of $N$. azteca Humbert \& Saussure, 1869; and a summary of the fauna (Scolopendromorpha: Scolopocryptopidae: Newportiinae). Zootaxa, 379(1): 1-20.

Cortés-Ríos, B.E. y J.D. Gárate-Rodríguez. 2017. Diversidad biológica de ciempiés en Surutato, Badiraguato, Sinaloa. Boletín de la Sociedad Mexicana de Entomología (n. s.), (3): 23-26.

Cupul-Magaña, F.G. 2009. Nuevas localidades para quilópodos (Chilopoda) en la costa de Jalisco y Sinaloa, México. Dugesiana, 16(2): 81-85.

Cupul-Magaña, F.G. 2013. La diversidad de los ciempiés (Chilopoda) de México. Dugesiana, 20(1): 17-41.

Cupul-Magaña, F.G. 2014. Registro de las especies de ciempiés (Chilopoda) y milpiés (Diplopoda) de Sinaloa, México. Actualidades Biológicas, 36(100): 73-76.

Cupul-Magaña, F.G. 2019. Diversidad de ciempiés de México(Myriapoda: Chilopoda).(pp: 50). In:Asociación Colombiana de Zoología (Ed.). Reconciliarte con la biodiversidad, un acto de paz. Libro de resúmenes. $V$ Congreso Colombiano de Zoología. Asociación
Colombiana de Zoología, Bogotá.

Cupul-Magaña, F.G. y J. Bueno-Villegas. 2016. Ciempiés (Chilopoda) y milpiés (Diplopoda) depositados en la Colección de Aracnología del Centro de Investigaciones Biológicas del Noroeste, A. C., La Paz, México. Boletín del Museo de Entomología de la Universidad del Valle, 17(1): 17-20.

Cupul-Magaña, F.G. and R.M. Shelley. 2011. A second locality in Jalisco, México, for the centipede Ectonocryptoides quadrimeropus Shelley \& Mercurio, 2005 (Scolopendromorpha: Scolopocryptopidae: Ectonocryptopinae). Entomological News, 122(4): 378379.

Cupul-Magaña, F.G., J. Bueno-Villegas, U.S. FloresGuerrero y E. Rodríguez-López. 2015. Ciempiés (Myriapoda: Chilopoda) depositados en la Colección Nacional de Arácnidos (CNAN) del Instituto de Biología, UNAM, México: registros recientes. Boletín de la Sociedad Entomológica Aragonesa (S.E.A.), (56): 364-366.

Dyachkov, Y.V. 2018. New data on the distribution of Pachymerium ferrugineum (C.L. Koch, 1835) (Chilopoda: Geophilomorpha: Geophilidae) in Central Asia. Ukrainian Journal of Ecology, 8(4): 252-254.

Edgecombe, G.D. y F.G. Cupul-Magaña. 2008. Primer registro de Scutigera linceci (Wood, 1867) para Jalisco y anotaciones sobre los escutigeromorfos de México (Chilopoda: Scutigeromorpha: Scutigeridae). Dugesiana, 15(1): 17-19.

Edgecombe, G.D. and L. Bonato. 2011. Order Scolopendromorpha. (pp. 392-407). In: Minelli, A. (Ed.). The Myriapoda: Treatise on zoology - anatomy, taxonomy, biology. Volume 1. Brill, Leiden.

Eisner, T., M. Eisner and M. Siegler. 2005. Secret weapons: defenses of insects, spiders, scorpions, and other many-legged creatures. The Belknap Press of Harvard University Press, Cambridge.

Flores-Guerrero, U.S., F.G. Cupul-Magaña, J. BuenoVillegas y E. Rodríguez-López. 2015. Adenda y corrigenda de Dugesiana 20(1): 17-41, la diversidad de los ciempiés (Chilopoda) de México. Dugesiana, 22(2): 69-80.

Folly, H., R. Thaler, G. Bard Adams y E. Almeida Pereira. 2019. Predation on Scinax fuscovarius (Anura, Hylidae) by Scolopendra sp. (Chilopoda: Scholopendridae) in the State of Tocantins, central Brazil. Revista Latinoamericana de Herpetología, 2(1): 39-43.

Lewis, J.G.E. 1981. The biology of centipedes. Cambridge University Press, Cambridge.

Martínez-Muñoz, C.A. y T. Tcherva. 2017. Primer registro de Newportia stolli (Pocock, 1896) (Chilopoda: Scolopendromorpha: Scolopocryptopidae) para Cuba y Las Antillas. Boletín de la Sociedad Entomológica Aragonesa (S.E.A.), (60): 179-184.

Minelli, A. 2011. The Chilopoda - Introduction. (pp. 2142). In: Minelli, A. (Ed.). The Myriapoda: Treatise on zoology-anatomy, taxonomy, biology. Volume 1. Brill, Leiden.

Mitić, B.M. and V.T. Tomić, 2002. On the fauna of centipedes (Chilopoda, Myriapoda) inhabiting Serbia and Montenegro. Archives of Biological Sciences, Belgrade, 54(3-4): 133-140. 
Molinari, J., E.E. Gutiérrez, A. Ascenção, J.M. Nassar, A. Arends and R.J. Márquez. 2005. Predation by giant centipedes, Scolopendra gigantea, on three species of bats in a Venezuelan cave. Caribbean Journal of Science, 41(2): 340-346.

Mundel, P. 1981. A review of the lithobiomorph centipedes of Mexico. Ph.D. Thesis. University of WisconsinMadison, Madison.

Pocock, R.I. 1895-1910. Chilopoda and Diplopoda. Biologia Centrali-Americana, 14: 1-217.

Ramamoorthy, T.P., R. Bye, A. Lot y J. Fa. 1998. Introducción. (pp.xxi-xxxi). In: Ramamoorthy, T.P., R. Bye, A. Lot y J. Fa (Eds.). Diversidad biológica de México: orígenes y distribución. Instituto de Biología, Universidad Nacional Autónoma de México, México, D.F.

Ribera, I., A. Melic y A. Torralba. 2015. Introducción y guía visual de los artrópodos. Revista Ide@-SEA, (2): 1-30.

Schileyko, A.A. 2002. Scolopendromorpha. (pp. 479-500). In: Adis, J. (Ed.). Amazonian Arachnida and Myriapoda. Pensoft, Sofia-Moscow.

Schileyko, A.A. 2013. A new species of Newportia Gervais, 1847 from Puerto Rico, with a revised key to the species of the genus (Chilopoda, Scolopendromorpha, Scolopocryptopidae). ZooKeys, (276): 39-54.

Schileyko, A. and A. Minelli. 1998. On the genus Newportia Gervais, 1847 (Chilopoda: Scolopendromorpha: Newportiidae). Arthopoda Selecta, 7(4): 265-299.

Recibido: 15 de marzo 2019

Aceptado: 31 de mayo 2019

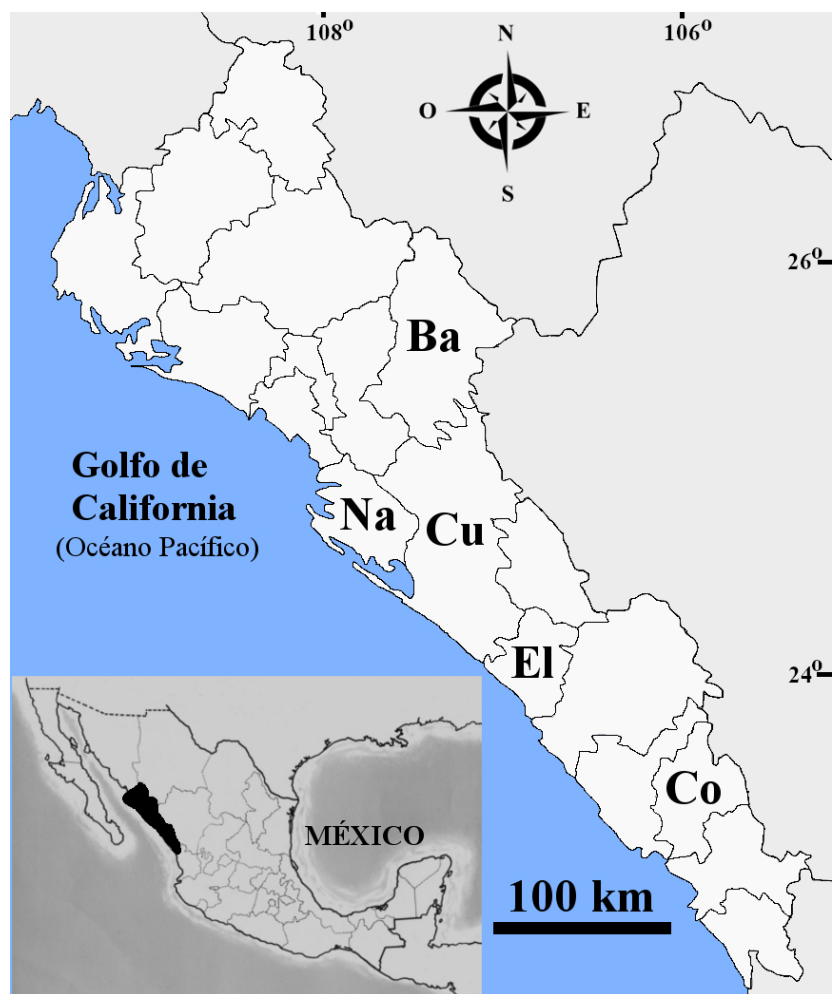

Shelley, R.M. 2002. A synopsis of the North American centipedes of the order Scolopendromorpha (Chilopoda). Virginia Museum of Natural History Memoir, (5): 1-105.

Shelley, R.M. 2006. A chronological catalog of the New World species of Scolopendra L., 1758 (Chilopoda: Scolopendromorpha: Scolopendridae). Zootaxa, (1253): $1-50$.

Shelley, R.M. 2009. Occurrence of the centipede Ectonocryptoides quadrimeropus Shelley and Mercurio, 2005, in central Mexico (Scolopendromorpha: Scolopocryptopidae: Ectonocryptopinae). Western North American Naturalist, 69(1): 138-139.

Shelley, R.M. and A. Chagas-Jr. 2004. The centipede genus Arthrorhabdus Pocock, 1891, in the western hemisphere: potential occurrence of $A$. pygmaeus (Pocock, 1895) in Belize (Scolopendromorpha: Scolopendridae: Scolopendrinae). Western North American Naturalist, 64(4): 532-537.

Shelley, R.M. and R. Mercurio. 2005. Ectonocryptoides quadrimeropus, a new centipede genus and species from Jalisco, Mexico; proposal of Ectonocryptopinae, analysis of subfamilial relationships, and a key to subfamilies and genera of the Scolopocryptopidae (Scolopendromorpha). Zootaxa, (1094): 25-40.

Shelley, R.M., G. Edwards and A. Chagas-Jr. 2005. Introduction of the centipede Scolopendra morsitans L., 1758, into northeastern Florida, the first authentic North American record, and a review of its global occurrences (Scolopendromorpha: Scolopendridae: Scolopendrinae). Entomological News, 116(1): 39-58.

Tabullo-De Robles, J., M.C. Gutiérrez y F.G. CupulMagaña. 2014. Nuevos registros de ciempiés (Myriapoda; Chilopoda) para Morelos, México. Acta Zoológica Mexicana (n. s.), 30(3): 731-733.

Verhoeff, K. 1934. Beiträge zur Systematik und Geographie der Chilopoden. Zoologische Jahrbücher, Abteilung für Systematik, (66): 1-112.

Voigtländer, K. 2011. Chilopoda - Ecology. (pp. 309325). In: A. Minelli (Ed.). The Myriapoda: Treatise on zoology-anatomy, taxonomy, biology. Volume 1. Brill, Leiden.

Würmli, M. 1973. Die Scutigeromorpha (Chilopoda) von Costa Rica. Ueber Dendrotherehua arborum Verhoeff, 1944. Studies on the Neotropical Fauna, (8): 75-80.

Figura 1. Municipios del estado de Sinaloa, México, con nuevos registros de localidades y especies de ciempiés. $\mathrm{Ba}=$ Badiraguato, $\mathrm{Co}=$ Concordia, $\mathrm{Cu}=$ Culiacán, $\mathrm{El}=$ Elota y $\mathrm{Na}=$ Navolato . 


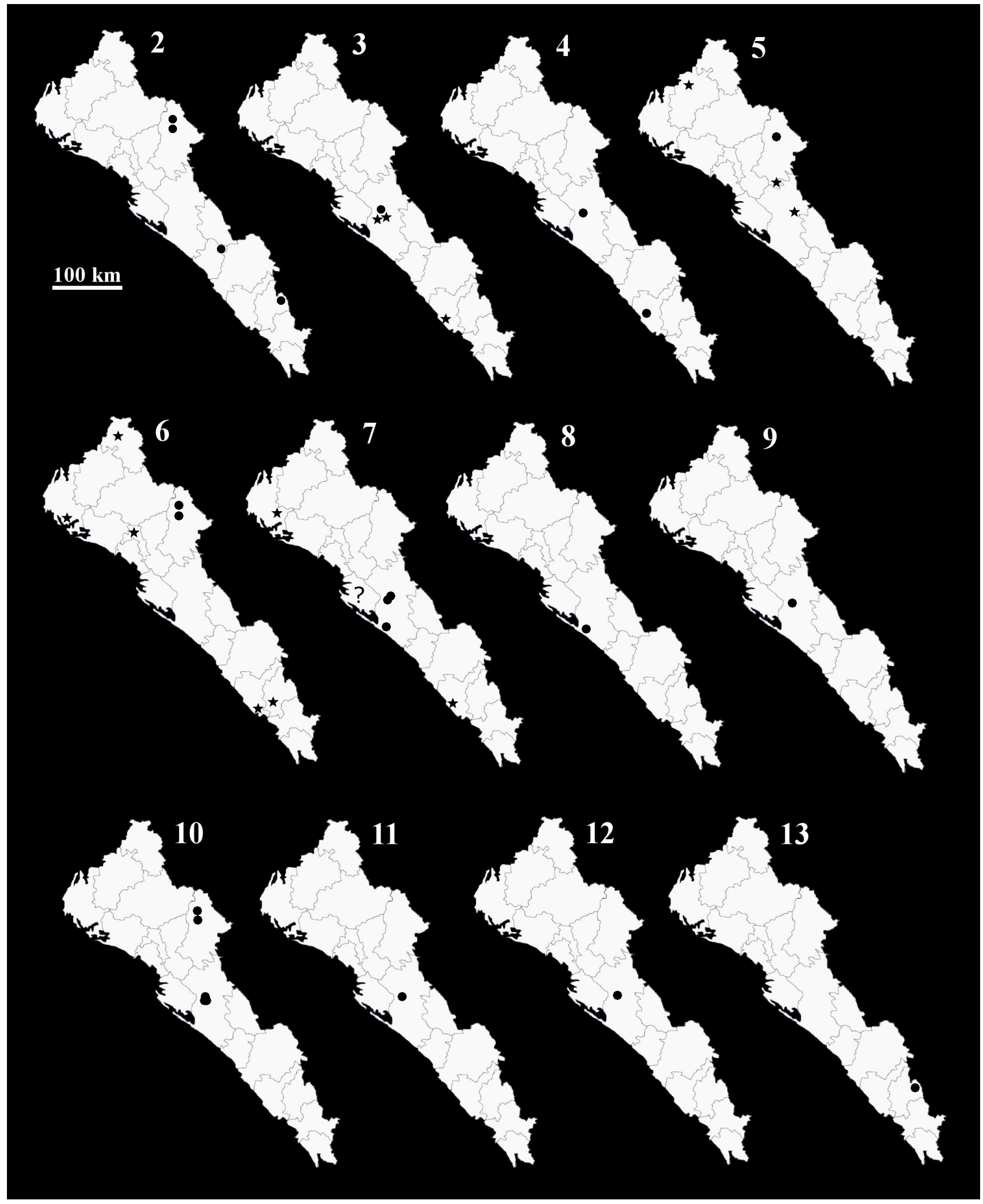

Figura 2-13. Localidades de registro de ciempiés en Sinaloa, México. Punto negro= nuevo registro, Estrella negra $=$ registro previo publicado, ? = sin localidad específica dentro del municipio. 2) Dendrothereua linceci. 3) Arthrorhabdus pygmaeus. 4) Scolopendra morsitants. 5) Scolopendra polymorpha. 6) Scolopendra viridis. 7) Rhysida longipes. 8) Newportia quadrimeropus. 9) Newportia stolli. 10) Pachymerium ferrugineum. 11) Mecistocephalus guildingii. 12) Notiphilides maximiliani. 13) Orphnaeus brevilabiatus. 